\title{
Bone marrow evaluation in patients with fever of unknown origin
}

\author{
Jha $\mathrm{A}^{1}$, Adhikari $\mathrm{RC}^{1}$, Sarda $\mathrm{R}^{2}$
}

${ }^{1}$ Department of Pathology, Institute of Medicine, Tribhuvan University, Kathmandu, Nepal

${ }^{2}$ Clinical Assistant Professor, Department of Pathology, University of Wiscosnin Hospital and Clinics, 600 Highland Ave, Madison, Wisconsin, USA

\author{
Keywords: \\ Bone marrow; \\ Culture; \\ Fever of unknown origin; \\ Neoplasm
}

\begin{abstract}
Bone marrow examination is commonly requested investigations in cases of fever of unknown origin, irrespective of alteration in hematological parameters. More than 200 etiologies are associated with fever of unknown origin, and they are broadly divided into infectious, neoplastic, collagen vascular diseases, miscellaneous and undiagnosed. Many of these conditions directly or indirectly affect bone marrow. Marrow may show changes in cellular components, interstitium or in the blood vessels depending on the underlying local or systemic conditions. The three main hematopoietic cell lines may show variable hyperplasia, hypoplasia or aplasia of one or more than one cell lines, and occasionally dyspoiesis. Interstitium may show fibrosis, gelatinous transformation, or infiltration by abnormal cells. Amyloid deposits may be seen around blood vessels. Marrow may also show granulomas, infectious agents or neoplastic cells. Various reactive changes can be seen in the bone marrow in neoplastic, infectious and in connective tissue diseases. Infectious agents can be cultured from the marrow aspirate or can be demonstrated in marrow.
\end{abstract}

\section{INTRODUCTION}

Fever of Unknown Origin (FUO) means fever that does not resolve spontaneously in the period expected for self-limited infection and the cause of which cannot be ascertained despite considerable diagnostic effort. Reported cases of FUO exceed 200, and fall into diverse subspecialty categories. There are no algorithms and few clues that reliably suggest or exclude particular diagnoses. ${ }^{1}$ The diagnostic spectrum of FUO is changing over time and FUO remains the diagnostician's challenge. ${ }^{2}$

\section{Correspondence:}

Dr. Abhimanyu Jha, $M D$

Associate Professor, Department of Pathology, Institute of Medicine,

Tribhuvan University, Kathmandu Nepal

Email: jhaabhimanyu@yahoo.com
Bone marrow examination (BME) is commonly undertaken as part of blind investigation of FUO. ${ }^{3}$ Bone marrow culture (BMCs) can be done at the same time and is especially helpful in disseminated bacterial, fungal and tuberculous infection. ${ }^{4}$ BMCs and blood cultures (BCs) are frequently obtained in the evaluation of FUO. However, the low yield of clinically significant isolates leads to question about their cost-effectiveness. BMCs are particularly useful for patients who are immuno-compromised. ${ }^{5}$ The response of the bone marrow varies, depending upon infectious and noninfectious causes. Bone marrow modifications resulting from infection and systemic disease can be studied by analysis of morphology and etiology, and can impart great influence in the management of patients with fever. 


\section{Fever of Unknown Origin (FUO)}

The most widely used definition of FUO was given by Petersdorf and Beeson in 1961 in a paper describing results of a study which they carried out in 100 patients. They defined FUO as temperature of $38.3^{\circ} \mathrm{C}\left(101^{\circ} \mathrm{F}\right)$ or above persisting or recurring during a period of two weeks and seven days investigation in hospital, or if three outpatient visits fail to result in a diagnosis. ${ }^{3,6}$

The criteria proposed by Petersdorf have been in use internationally to define the FUO since 1961 and the research of FUO has progressed with this criterion. Meanwhile, new diagnostic methods have been developed and illness behavior of febrile patients has changed considerably. Kohno-H and colleagues have developed a new criterion of FUO. According to this criteria, FUO constitutes, outpatients who are febrile for more than two weeks, documented temperature higher than $37.5^{\circ} \mathrm{C}$ at least on one occasion and undetermined diagnosis and inpatients who are febrile for more than one week with documented temperature higher than $37.5^{\circ} \mathrm{C}$, and undetermined diagnosis. ${ }^{7}$

Over last 40 years, health care system has shifted from the inpatient to the ambulatory setting. As a result it has now become widely accepted that requirement for one week evaluation in hospital be modified so that evaluations may now be completed in an outpatient setting. ${ }^{2}$ With the advent of immunosuppressive therapies and emergence of AIDS, it has been suggested that the requirement for in-hospital investigation is deleted, and further define patients with FUO based upon the setting and whether or not the patient is immunocompromised. This approach permits a more targeted series of investigations based upon the disorders that are more likely to be prevalent in the host populations. ${ }^{8}$ In 1991, Durack and Street proposed a new system for the classification of FUO: classic FUO in non-immunocompromised patients, nosocomial FUO, neutropenic FUO, and FUO associated with HIV infection. ${ }^{8}$ In addition there are factitious fever, essential hyperthermia, fabricated fever, drug induced and occupational FUO which are considered as clinically benign FUO ${ }^{1,3,4,9}$ The diagnostic and therapeutic approach in immunocompromised patients is conceptually different from that in patients with "classic" FUO and is not dealt with specifically in this review.

\section{Causes of FUO}

Over 200 separate diseases have been reported to produce prolonged fever or recurrent febrile episodes, which have defied easy diagnosis. ${ }^{1}$ Despite the large number almost all can be classified into five categories proposed by Petersdorf and Beeson: infectious, neoplasms, collagen vascular disease, miscellaneous, and undiagnosed when cause is not identified. The proportion of patients in each category varies depending upon geographical distribution, age group under consideration, the duration of febrile disorder and presence or absence of underlying disorder. ${ }^{10,11}$ Infection remains the leading cause of FUO in most series. Among the infections tuberculosis leads the list. Fresh resurgence of malaria and kala-azar is a formidable challenge. Neoplasms account for the next common cause followed by collagen vascular disease and miscellaneous. The cause of the fever in malignant disease is unknown. Endogenous pyrogens are possibly liberated from the cells of mononuclear phagocyte series in the liver or bone marrow. Tumor cells have also been shown to produce pyrogens. The nature of cytokine responsible, is not clear but tumor necrosis factor (TNF) and interleukin-2 (IL-2) may be produced in cancer patients. ${ }^{12}$ Fever in neoplastic conditions, collagen vascular diseases and in some infections can last for several months. ${ }^{13}$ Collagen vascular disease ranges from Still disease and juvenile rheumatoid arthritis in younger patients to giant cell arteritis or polymyalgia rheumatica in elderly. Infection, neoplasm and inflammatory disease contribute about 60 to $70 \%$ of FUO. They contribute only $20 \%$ of cases of episodic or recurrent FUO; miscellaneous groups of diseases are major cause of episodic FUO.9 The list of some the causes of FUO is shown in table 1.1,14

\section{Role of bone marrow examination in FUO}

BME is one of the oldest and most valuable diagnostic tools in the assessment of hematological disorders. The relative ease of the bone marrow trephine biopsy procedure has led to its routine use in the diagnosis of infections, hematological disorders, metastatic tumors, and metabolic disorders. Bone marrow aspiration is an old and wellestablished test procedure, the use of which may rest more on diagnostic tradition than on scientific documentation. ${ }^{15}$ Its use was evaluated in a Norwegian regional hospital by registering indication, presence of pathology and clinical "utility" judged from a given definition, in 200 reports on bone marrow aspiration. The result was pathological in $53 \%$, and judged to be clinically useful in $61 \%$ of the tests. The diagnostic yield was highest for indications related to bone marrow function and low for nonspecific indications such as FUO and elevated ESR without M-component. The large numbers of results with pathological findings that are judged to be clinically useful raise the question of whether use of this test should be increased. BME including biopsy is useful in the investigation of FUO as it leads to an etiological diagnosis in most cases. ${ }^{16}$ As a screening procedure this investigation is of little use and anemia alone is certainly not a reason to perform this investigation in patients with FUO ${ }^{17}$ In up to $15 \%$ of FUO cases, bone marrow aspiration and biopsies have been useful in providing specific diagnostic information in absence of hematologic or liver function abnormalities or hepatic enlargement. ${ }^{6}$ A combined use of bone marrow aspiration, biopsy and culture helps in the rapid diagnosis of mycobacterial and histoplasma infections in HIV patients. ${ }^{18}$ In patients with FUO BME is often performed for indications related to hematological conditions, infections and malignancies. 


\section{Hematological conditions}

BME is carried out if any hematological parameter is abnormal. It may help in diagnosing a hematological malignancy, granulomatous disorder or parasitic diseases. Bone marrow biopsy is likely to show granuloma in only half of the cases, but yield exceeds $80 \%$ when anemia, leucopenia and monocytosis are present. ${ }^{1}$

In patients with FUO accompanied by pancytopenia, BME constitutes an important test either in confirming the diagnosis or in excluding a primary marrow involvement and suggesting alternative investigations for diseases like SLE and hypersplenism. ${ }^{19}$ Bone marrow aspirate specimens are superior for morphological details over biopsy, while biopsy specimens provide a more reliable index of cellularity and often reveal presence of various lymphomas, marrow infiltration, fibrosis, and granulomas which are not detected in aspiration. ${ }^{20}$ Both bone marrow biopsy and aspiration should be performed simultaneously. In cases of associated pancytopenia BME can reveal tuberculosis, myelodysplastic syndrome, acute leukemia, HIV infection, kala-azar, aplastic/ hypoplastic anemia and megaloblastic anemia. ${ }^{21}$ Bone marrow aspiration in case of FUO related to Vitamin B12 deficiency can reveal diagnosis of megaloblastic anemia. ${ }^{22}$

\section{Infections}

In case of disseminated tuberculosis, histoplasmosis, and brucellosis bone marrow biopsy may reveal granuloma and the organisms. ${ }^{1,20}$ Various hematological manifestations in patients with tuberculosis can be studied by BME. Pancytopenia in peripheral blood is observed in patients with disseminated/ miliary tuberculosis. Thrombocytosis is more common in patients with pulmonary tuberculosis while thrombocytopenia is common in patients with disseminated/ miliary tuberculosis. Hemophagocytosis, plasmacytosis, necrosis, and granuloma can be observed in bone marrow of patients with tuberculosis. ${ }^{23}$ Pancytopenia secondary to infection can be confirmed by bone marrow aspiration and biopsy that reveals fatty material with diffuse sparse accumulation of lymphocytes, phagocytizing macrophages, conspicuous mast cells, and plasma cells. Normal hematopoietic precursors are scarce and often greatly dispersed. ${ }^{24}$ In immunocompromised patients with generalized cytomegalovirus (CMV) infection, BME might reveal marrow suppression, infiltration of marrow with lymphocytes and macrophages. The macrophages may exhibit hemophagocytosis. Bone marrow studies when indicated can be of great help in establishment of diagnosis because of the hematological alterations in infectious diseases. ${ }^{24}$ Bone marrow sampling has diagnostic utility in HIV infected patients with pyrexia without localizing signs, pancytopenia, and staging/investigation of lymphoma. It can reveal unexpected lymphoma, mycobacteriosis, and toxoplasmosis. ${ }^{25}$
Bone marrow biopsy is a useful procedure for the diagnosis of fever in patients with advanced HIV disease, particularly in areas where tuberculosis and leishmaniasis are prevalent. Involvement of the marrow may be the first indication of the existence of extranodal Non-Hodgkin lymphoma (NHL). ${ }^{26}$

Parvovirus B19 causes hematological disease mainly in hemolytic anemia patients because of its tendency to infect rapidly proliferating cells. FUO in Parvovirus B19 infection is well known. It can cause pure red cell aplasia or transient aplastic crisis in such patients. ${ }^{24}$

\section{Malignancies}

BME is a part of investigation of hematological malignancies and bone marrow trephine biopsy should be a routine part of investigation in patient with bone marrow failure and will be of particular value in the diagnosis of those with disseminated malignancies.

Bone marrow aspirates and trephine biopsies are complementary procedures and both are required for diagnosis, classification and decisions on current treatment modalities of patient with Myelodysplastic syndromes (MDS). Three types of abnormalities are observed in the bone marrow (1) cellular abnormalities, (2) architectural disorganization in the bone marrow and (3) stromal changes. ${ }^{27}$ Bone marrow biopsy is a useful procedure in diagnosis, subclassification, and staging of lymphoproliferative disorders and diagnosis of plasma cell dyscrasias. Metastatic tumors to bone marrow are easily detected in aspiration as well as bone marrow biopsy.

\section{Role Of Bone Marrow Culture (BMC)}

BMC constitutes an important investigation in patients with FUO. BMCs can be positive when BCs are negative. ${ }^{28}$ In patients with sub-acute bacterial endocarditis who have received prior antibiotics, the incidence of positive $\mathrm{BMC}$ exceeds that of $\mathrm{BC} .^{20}$

Enteric fever is the only bacterial infection of humans for which BMC is routinely recommended. It is generally believed the BMC will increase the diagnostic yield by one third compared with that from BC. The viable organism counts in the bone marrow is considerably less affected by antibiotic treatment than blood counts. ${ }^{29}$ Mycobacterium avium complex (MAC), Mycobacterium tuberculosis, and Histoplasma capsulatum infections are common in patients with AIDS. BCs, however, are more sensitive than BMC in detecting these organisms. ${ }^{18,26,30}$ Morphological examination of bone marrow combined with cultures of marrow is useful in diagnosing disseminated histoplasmosis in patients with AIDS. BMC is more sensitive than Ziehl-Neelsen (Z$\mathrm{N})$ staining in demonstration of marrow mycobacteria. ${ }^{31}$ BMCs for Brucella are recommended for patients with FUO for whom the routine workup turns out to be negative. 
Serological tests for brucellosis can be false-negative in some cases of brucellosis due to prozone phenomena. ${ }^{32}$

BMCs are probably not justified as part of the routine initial evaluation of patients with FUO, regardless of immunological status, given the cost, the low yield of clinically significant isolates, and the comparable $\mathrm{BC}$ results. ${ }^{5}$

BMC for Leishmania donovani on NNN (Novy, MacNeal and Nicolle) media may be helpful, particularly in early stage of kala-azar when spleen is not sufficiently enlarged as to be punctured for culture. Compared to splenic puncture it is safer procedure. Promastigote forms of Leishmania donovani are demonstrated in culture. However, culture of leishmania on NNN media is time consuming. ${ }^{33}$ Penicillium marneffei a dimorphic fungus that can infect immunocompromised patients can be cultured in blood or bone marrow.

\section{Detection of organisms in bone marrow}

Direct examination of marrow under microscope can be used to identify bacteria, fungi, and parasites. Routine staining of bone marrow can demonstrate some parasite and fungi. Special stains like Z-N and Gram can be done to identify mycobacterium and other bacteria in suspected infective origin. Z-N stain for AFB should be performed routinely in marrow of patients with AIDS, as organisms can be detected in marrow irrespective of presence of granulomas.

Detection of protozoa like malarial parasite, amastigote form of leishmania (LD bodies), trypanosoma are well known but detection of microfilaria are also reported in bone marrow aspirate smears. ${ }^{34}$ Tropheryma whippelli (Whipple disease) may be seen inside the bone marrow macrophages. Bacteria visible inside macrophage have also been reported in bacterial endocarditis. ${ }^{35}$

Fungi are rarely seen in bone marrow aspiration except in immunocompromised patients such as HIV infection or following bone marrow transplantation or cancer chemotherapy. ${ }^{35}$ Organisms may be detected within macrophages, megakaryocytes, neutrophils. Toxoplasma gondii (free forms and pseudocysts) can be identified in the cytoplasm of macrophages, granulocytes and within megakaryocytes in bone marrow biopsy specimens of patients with AIDS. Bone marrow biopsy plays an important role in the identification of such agents in patients with unexplained fever and particularly in HIV positive patients. ${ }^{36}$

\section{Bone marrow morphology}

It is now thought that cytokines, principally, IL-I and TNF produced by leukocytes in response to infectious agents or to immune and toxic reactions are involved in the origin of fever. ${ }^{13}$ Bone marrow responds to inflammation by accelerated release of cells from post mitotic reserve pool caused by IL-I \& TNF and is associated with an increase in the number of more immature neutrophils in blood and sometimes causing extreme elevation of leukocytes in the peripheral blood leading to leukemoid reaction. ${ }^{35}$ Prolonged infection also induces proliferation of precursors in the bone marrow caused by increased production of cytokines. However, certain infections such as typhoid fever, viral infection, rickettsial \& protozoal infections cause suppression of bone marrow leading to leukopenia. ${ }^{35}$

\section{Morphological alterations in bone marrow cytology and histology}

There are various morphological alterations in bone marrow cytology which is evaluated in bone marrow aspiration smears and architectural alterations are evaluated in trephine biopsy. The changes in cytology and histology both in inflammatory as well as neoplastic diseases are described below:

\section{Bone marrow manifestation in inflammatory diseases}

The bone marrow response to inflammation depends on nature and chronicity of the infection/inflammation, the age of the subject, presence of any associated diseases, and whether the infection is bacterial, viral, rickettsial or fungal. Morphologically, similar lesions can arise from different pathological agents and one disease can cause several different lesions.

\section{Bone marrow cytological manifestations}

In severe bacterial infections the marrow features reflects those of the peripheral blood. For example, the response may be granulocytic hyperplasia with depressed erythropoiesis, increased megakaryocytes and macrophages with hemophagocytosis. Erythroid hyperplasia can also occur in acute inflammation. In severe infections marrow hypoplasia as well as fibrin deposition, necrosis and even fibrosis can occur which are better studied in the trephine biopsy. ${ }^{36}$ Leukemoid reaction can occur in both acute and chronic inflammatory conditions. In addition marrow eosinophilia can occur in hypersensitivity reactions, collagen vascular diseases and parasitic infestations. ${ }^{36}$ In chronic infection there are often increased iron stores. Additionally, in tuberculosis, there are increased macrophages, increased iron stores. Bone marrow findings in cases of disseminated/ miliary tuberculosis and pulmonary tuberculosis include, reactive marrow (most cases), hypocellular marrow, megaloblastosis, necrosis, dyspoiesis (dysmyelopoiesis, dyserythropoiesis and dysmegakaryopoiesis), plasmacytosis, histiomonocytosis, lymphocytosis, histiohemophagocytosis, and altered myeloid-erythroid ratio. ${ }^{23}$ In addition there may be epithelioid granuloma in cases of bone marrow tuberculosis. In Brucellosis there 
is hypercellular marrow, hemophagocytosis, increased eosinophils and plasma cells. ${ }^{24}$ In enteric fever bone marrow may show variable degree of myeloid hyperplasia, lymphocytosis, and monocytosis. ${ }^{29}$

Marrow lymphocytosis is also seen in viral infections and may show increased atypical lymphocytes similar to peripheral blood smear findings. In various viral infections prominent hemophagocytosis can be evident in the marrow. ${ }^{36}$ Parvovirus B19 infection is responsible for hemophagocytic syndrome leading to aplastic crisis in both children and adults. ${ }^{36}$ There is thrombocytopenia due to increased destruction of platelet. Erythroid hyperplasia with giant erythroblasts and multinucleated erythroblasts can be seen in the marrow. Viral inclusions can be seen in the nuclei of erythroblasts. ${ }^{24,36,37}$ In virus associated hemophagocytic syndrome (VAHS) bone marrow shows hyperplasia of mature histiocytes with phagocytosis of red cells, platelets and nucleated hematopoietic cells. These histiocytes have low nuclear-cytoplasmic ratio, which should be distinguished from malignant histiocytosis. Malignant histiocytes are immature high nuclear-cytoplasmic ratio, large nuclei with prominent nucleoli. No phagocytosis is seen in malignant histiocytes. ${ }^{37}$ Although the term VAHS is used, bacteria, fungi and parasites may induce secondary hemophagocytic lymphohistiocytosis as well. ${ }^{36}$ Bone marrow in HIV infection may show variable cellularity, erythroid hyperplasia, megaloblastic hematopoiesis, megakaryocytic hypoplasia, eosinophilia, plasmacytosis, granuloma and gelatinous marrow transformation. Gelatinous transformation of bone marrow is also seen in other infections such as tuberculosis, infective endocarditis and pneumonia. ${ }^{36}$ Marrow plasmacytosis is common in HIV infection and iron studies show features of anemia of chronic disease. Marrow plasmacytosis can be seen in various bacterial infections, viral infections, dysimmune diseases, hyepersensitivity, malignant diseases, and metabolic diseases ${ }^{36}$ In patients with AIDS, H. capsulatum are seen within the marrow elements or within polymorphonuclear cells and seen only in marrow elements showing diffuse lymphohistiocytic aggregates. ${ }^{35}$

In malaria infection there are no specific cytological changes in the bone marrow. Marrow cellularity is variable and no significant alteration in erythropoiesis or myelopoiesis seen, however, parasites can be detected. In Visceral leishmaniasis marrow shows several changes: ${ }^{38}$

1. Presence of $L D$ bodies

2. Decreased myeloid-erythroid ratio indicating relative suppression of myelopoiesis

3. Increased number of plasma cells up to $10 \%$ indicating increased antibody formation.

4. Presence of giant metamyelocytes indicating suppression of cell division

5. Presence of juvenile megakaryocytes indicating increased formation of platelets to meet demand caused by increased destruction in hyperactive spleen.

6. Presence of micronormoblasts in aggregates indicating splenic hyperactivity.

7. Crenated LD bodies with deformed cell membranes in cases of treated kala-azar.

In cases of connective tissue disorder, marrow aspirate shows variable cellularity. Megaloblasic changes may be present and megakaryocytes may be increased or decreased. Some of the patients treated with cytotoxic agents may show features of myelodysplastic changes. Iron stores and myeloid series show variable abnormalities. ${ }^{35}$

\section{Bone Marrow Trephine Biopsy}

In bone marrow biopsy two types of modification can be observed. Those occurring in connective tissue comprising inflammatory processes, acute and chronic as well as immune reactions, and those involving normal hematopoietic cell lines, with possible hyperplastic or aplastic changes in one or more cell lines. The stroma and vessel reactions comprise myelofibrosis, gelatinous transformation or amyloid deposits. $^{36}$

\section{2 a. Histological changes in acute inflammation}

In acute inflammation, mainly four types of changes can be seen, they are- exudative, hemorrhagic, necrotic and suppurative type of changes.

Exudative type changes are characterized by interstitial edema, small hemorrhages, congestion and endothelial swelling. Stellate fibrin deposits between hematopoietic cells may be seen.$^{36}$ Edema needs to be distinguished from gelatinous transformation of marrow which is characterized by hyaline deposits between adipocytes in areas without any hematopoietic cells. This substance is faintly eosinophilic, Periodic Acid Schiff (PAS) positive and may show metachromasia with Giemsa stain. It is observed in varieties of conditions including severe protein deficiency malnutrition and HIV positive patients. ${ }^{36}$ Bone marrow edema is also a feature of disseminated toxoplasmosis in patient with AIDS and associated with necrosis and scattered or clusters of histiocytes.

In hemorrhagic type of marrow changes, in acute inflammatory diseases, the hemorrhage is often associated with hypoplasia of hematopoietic cells. Such features are seen in Hepatitis B infection or in patient with severe histiocytic activation syndrome. ${ }^{36}$

In necrotic type of changes, necrosis is often patchy interspersed between and adjacent to normal marrow. Associate fat atrophy and focal hypoplasia are frequently present.36 Bone marrow necrosis has been associated with prognosis in patients with serious hematologic diseases and metastatic carcinoma. Ischemic necrosis secondary 
to arteriolar thrombosis may be found in patient with septicemia, bacterial endocarditis, and different bacterial, viral, fungal or parasitic infection, Q-fever, dysimmune disorders such as disseminated lupus erythematosus, or hypersensitivity. Stellate fibrin deposits can be associated with necrosis. In some cases fibrosis replaces the necrotic areas. ${ }^{36}$ The necrosis of inflammatory origin should be distinguished from bone marrow necrosis that occurs in sickle cell anemia or drug toxicity. Antiphospholipid syndrome is also associated with extensive marrow necrosis and pancytopenia. ${ }^{36}$

Disseminated tuberculosis produces caseous necrosis surrounded by a few histiocytes without epithelioid cells. Such necrosis is present along bone trabeculae or in the adipose tissue. ${ }^{36}$ By Z-N stain AFB can be demonstrated in necrotic areas.

Suppurative type of marrow changes is seen in osteomyelitis and characterized by neutrophil hyperplasia away from the lesion and suppurative necrosis containing basophilic debris, neutrophils and macrophages. Marrow fibrosis, plasmacytosis and osteoblastic proliferation will follow. ${ }^{36}$

\section{2 b. Trephine biopsy changes in chronic inflammation and immune reactions}

Stromal reactions of chronic inflammation and immune reaction in bone marrow are characterized by (1) Granulomatous chronic inflammation; (2) Reactive lymphoid lesions; (3) Plasmacytosis; (4) Histiocytic hyperplasia; (5) Chronic inflammation with fibrosis; and (6) Chronic inflammation with amyloidosis. ${ }^{36,37}$ Many of these inflammatory reaction patterns are quite non-specific.

\section{2 b.1. Granulomatous chronic inflammation}

Granulomas are identified by an accumulation of epithelioid histiocytes with sharp outlines which may be associated with giant cells and variable number of lymphocytic infiltration. Different types of necrosis, according to etiology occupy the centers of granuloma e.g. fibrinoid necrosis in hypersensitivity; caseous necrosis in tuberculosis. ${ }^{36}$ Noncaseating granuloma may be associated with connective tissue disorders and sarcoidosis. Fibrosis may envelop the granulomas or even penetrate between the cells. Granulomas may be found in lacunar spaces or occasionally in close contact with bone trabeculae. Plasma cells and eosinophils often accumulate nearby. ${ }^{36,39}$ Important diseases causing granulomatous inflammation are listed in table $2 .^{36,39}$

In order to establish a relationship between infection and granuloma, the identification of a microorganism through a culture is a more reliable test. Definitive morphological characteristic which allows an etiologic diagnosis of bone marrow granulomas is difficult to ascertain. Epithelioid granulomas in marrow are seen in about $40 \%$ of patients with miliary tuberculosis. In AIDS patients small poorly formed granulomas are seen. $15-30 \%$ of patients with sarcoidosis have epithelioid granuloma in their bone marrow. An increased activity of osteoclast leads to increased bone resorption and hypercalcemia. ${ }^{36}$

In infectious mononucleosis, granulomas are smaller, less nodular and poorly delimited unlike in tuberculosis, sarcoidosis or brucellosis. In addition in Epstein-Barr Virus (EBV) infection dispersed erythrophagocytic histiocytes can be observed as well as small histiocytic granulomas around the adipocytes. Epithelioid granuloma can also be present in CMV and HIV infections with or without opportunistic infections. ${ }^{36}$

In patients with immunosupression, bone marrow granuloma is associated with infection with Mycobacterium avium intracellulare (MAI), Histoplasma capsulatum, ${ }^{31}$ and different strains of leishmania. Granuloma in cases of MAI infection composed of very small, ill defined collections of lymphocytes, histiocytes, and eosinophils to larger, denser lymphohistiocytic aggregates, and compact, easily recognized clusters of epithelioid histiocytes admixed with a few lymphocytes. Necrosis may be absent and giant cells are rare. ${ }^{4}$

Marrow granulomas can also be identified in autoimmune disease such as primary biliary cirrhosis, Crohn disease and rheumatoid arthritis. ${ }^{36}$

Epithelioid cells and/or histiocytic granulomas can be observed in NHL, leukemia, Hodgkin disease and in myeloproliferative disorders. It is worth remembering that they can be observed in absence of bone marrow involvement. Bone marrow granuloma may develop in various infiltrating carcinomas e.g. infiltrating lobular carcinoma of breast without light microscopic evidence of cancer in bone marrow. Cytokeratin, an immunohistochemical marker identifies small clusters of carcinomatous cells near the granuloma. ${ }^{36}$

In case of lesions with a great lymphocytic and vascular proliferation plus the presence of epithelioid cells and fibrosis, NHL with bone marrow involvement with a granulomatous pattern should be strongly considered. Granulomas may take variable appearance in NHL. It may be discrete focal to confluent granulomas replacing large areas of marrow or there may be lymphoid nodules with adjacent histiocytic cells with or without giant cells.

Bone marrow granuloma have also been described in association with certain drugs such as 2-cholorodioxyadenosine for the treatment of hairy cell leukemia, IL-2 for the treatment of acute myeloid leukemia, interferon-alpha for the treatment of chronic myeloid leukemia, and patients taking chlorpromazine or tocanide. ${ }^{36}$ 


\section{2 b.2. Reactive lymphoid lesions ${ }^{36}$}

Lymphoid cell hyperplasia has been described in numerous different diseases but with highly variable frequency. Three different types of reactive lymphoid lesions are observed and they should be distinguished from one another: i. reactive lymphocytes aggregates, ii. lymphoid follicles with germinal centers; and iii. reactive lymphohistiocytic infiltrates. ${ }^{36}$

i. Reactive lymphocytes aggregates: They are one to five in number and are composed of small to medium sized $\mathrm{T}$ or $\mathrm{B}$ lymphocytes. There may be a few histiocytes, plasma cells, mast cells and eosinophils. They are well circumscribed and well demarcated from the surrounding hematopoietic cells. They are seen in close contact with small blood vessels and are located away from trabeculae. Their significance is unknown and can be seen naturally in older individuals; however, they can also be seen in chronic inflammatory and dysimmune diseases. Sometimes they are associated with NHL and Hodgkin disease.

ii. Lymphoid follicles with germinal centers: They are one to two in number and show typical germinal centers with tangible body macrophages and have typical mantle zone. Germinal center may show mitotic activity and centrifollicular cell. Their location and significance are similar to reactive lymphocytes aggregates.

iii. Reactive lymphohistiocytic infiltrates: They are composed of mixed population of lymphoid cells at different stages of maturation, histiocytes that may be activated, mast cells, plasma cells and eosinophils. Scattered clusters of epithelioid cells can also be seen. They have poorly defined margin and can fill one or more medullary space. They do not show germinal center. This is not seen in normal individuals even in older age. They can be seen in viral diseases, initial period of HIV infections or in dysimmune diseases. The lesion is sometimes difficult to distinguish from NHL and Hodgkin disease.

\section{2 b.3. Plasmacytosis}

It is a condition in which plasma cells of mature type are present along capillaries and seen in patients with chronic inflammatory diseases, in dysimmune disorders such as Castleman disease, in septicemia and in HIV infection. ${ }^{36}$ They are rarely present in close contact with trabeculae. It should be distinguished from atypical and giant plasma cells of multiple myeloma. Presence of such plasmacytosis is very helpful for the diagnosis of reactive diseases. They are seen in infectious diseases (bacterial, protozoal such as leishmania and viral such as EBV, HIV), dysimmune disorders (Castleman disease, Hemolytic anemias, ITP, Collagen vascular diseases, Hypersensitivity, Monoclonal gammopathy of uncertain significance, immune amyloidosis.), malignant diseases even without bone marrow involvement (Hodgkin disease, NHL, CML), various other conditions (Iron deficiency anemia, Megaloblastic anemia, Cirrhosis, and Hypoplasia or Aplasia). ${ }^{36}$

\section{2 b.4. Histiocytic hyperplasia}

Histiocytic hyperplasia can be disseminated in the marrow dispersed between hematopoietic cells. They can contain iron granules or phagocytose infectious agents such as leishmania, histoplasma or even mycobacteria and can be demonstrated by special stains. ${ }^{36}$ Histiocytosis can also be associated with hemophagocytic syndrome in which marrow biopsy shows increase in number of histiocytes and they are disseminated throughout the marrow or present as small clusters of macrophages. Erythrocytes are seen in the cytoplasm of large histiocytes. Occasionally lymphocytes, granulocytes or erythroblasts are also phagocytosed. Reactive lymphoid aggregates, polymorphic lymphoid lesions or follicles with germinal centers may also be associated. ${ }^{36}$ In early stage, bone marrow is hypercellular and erythrophagocytosis is rare. In more advanced stages, marrow is hypocellular, with reduced erythropoiesis and granulopoiesis. The number of megakaryocytes can be normal or increased. Often there are alternate hypocellular and cellular areas. Areas of bone marrow destruction due to focal edema, hemorrhage and necrosis with depletion of hematopoietic cells can be observed. Myelofibrosis can develop particularly in areas of necrosis. Hemosiderin laden macrophages can be observed in fibrotic areas. In such situation viral inclusions should be looked for and lymphomatous infiltration should be ruled out. ${ }^{36}$ Some of the causes of erythrophagocytic syndrome are: ${ }^{36,37}$

- Infections such as bacterial (e.g.Salmonella typhi, Escherichia coli, Brucella species, Mycobacterium tuberculosis, Legionella, etc.), and viruses (Herpes virus, Adenovirus, Measles virus, Parainfluenza virus, Vaccinia virus, Rubella virus, HIV, Epstein Barr virus, CMV and Rubella), fungi (Histoplasma), protozoa (Toxoplasma, Leishmania and Malaria).

- Malignant diseases such as malignant lymphomas, Hodgkin disease and disseminated carcinoma.

- Famillial hemophagocytic lymphohistiocytosis

- Immune deficiency such as acquired viral diseases, immunosuppressive drug therapy and organ transplant.

\section{2 b.5. Chronic inflammation with fibrosis}

In various chronic inflammatory conditions such as tuberculosis and dysimmune disease, fibrosis and/or granulomas develop around the vessels. Fibrosis also develops at the areas of necrosis. There are associated plasma cells and lymphocyte infiltration. Lymphoid nodules may develop in contact with fibrosis. Mast cells and eosinophils can be increased in number in areas of fibrosis. ${ }^{36}$ However, these changes are nonspecific. 
Table 1. Causes of FUO $\mathbf{O}^{1,14}$

$\begin{array}{ll}\text { Infection } & \text { Intraabdominal abscess (eg periappendiceal, diverticular, subphrenic); liver, splenic, pancreatic, psoas or placental } \\ \text { abscess,Appendicitis, cholecystitis, cholangitis, aortoenteric fistula, mesenteric lymphadenitis, tuboovarian abscess, pyometra, } \\ \text { intracranial abscess, sinusitis, mastoiditis, otitis media, dental abscess, Chronic pharyngitis, tracheobronchitis, lung abscess } \\ \text { Septic jugular phlebitis, mycotic aneurysm, endocarditis, intravenous catheter infection, vascular graft infection, Wound } \\ \text { infection, osteomyelitis, infected joint prosthesis, pyelonephritis, and prostatitis, Tuberculosis, Mycobacterium avium complex, } \\ \text { leprosy, Lyme disease, relapsing fever (Borrelia recurrentis), syphilis, Q fever, legionellosis, yersiniosis, Salmonellosis } \\ \text { (including } \\ \text { Typhoid fever), listeriosis, Campylobacter, brucellosis, tularemia, bartonellosis, ehlichiosis, psittacosis, Chlamydia pneumoniae, } \\ \text { murine typhus, scrub typhus, Gonococcaemia, Meningococcaemia, Actinomycosis, nocardiosis, melioidosis, Whipple's disease } \\ \text { (Tropheryma whippelli), Candidaemia, cryptococcosis, histoplasmosis, coccodiomycosis, blastomycosis, sporotrichosis, } \\ \text { aspergillosis, mucormycosis, Malassezia furfur, Pneumocystis carini, Visceral leishmaniasis, malaria, babesiosis, toxoplasmosis, } \\ \text { schistosomiasis, fascioliasis, toxocariasis, amoebiasis, infected hydatid cyst, trichinosis, trypnosomiasis, Cytomegalovirus, } \\ \text { HIV, Herpes simplex, Epstein-Barr virus, parvovirus B19 }\end{array}$

Neoplasia

Collagen vascular

disease

Miscellaneous

All common malignant diseases including MDS

Adult still disease, SLE, cryoglobulinaemia, Reiter's syndrome, rheumatic fever, giant cell arteritis/ polymyalgia rheumatica, Wegner's granulomatosis, ankylosing spondylitis, Behecet's syndrome, polyarteritis nodosa, Hypersensitivity vasculitis, utricarial vasculitis, sjogren's syndrome, polymyositis, rheumatoid arthritis, erythema multiforme, erythema nodosum, relapsing polychondritis, mixed- connective tissue disease, Takayasu aortitis, Weber-Christian disease, Felty's syndrome, eosinophilic fascitis.

\section{Hematoma, thrombosis, recurrent pulmonary embolism}

Aortic dissection, femoral aneurysm, post-myocardial infarction syndrome, atrial myxoma, Sweet's syndrome, familial Mediterranean fever, familial Hibernian fever, hyperimmunoglobulin D syndrome, Crohn disease, ulcerative colitis, sarcoidosis, granulomatous hepatitis, subacute (de Quervain's) thyroiditis, hyperthyroidism, adrenal insufficiency, primary hyperparathyroidism, hypothalamic hypopituitorism, autoimmune hemolytic anemia, gout, pseudogout, Cirrhosis, chronic active hepatitis, alcoholic hepatitis, shunt nephritis, malacoplakia, Kawasaki's syndrome, kikuchi's Syndrome, mesenteric fibromatosis, inflammatory pseudotumour, Castleman's disease, Vogt-Koyanagi-Harada syndrome, Gaucher disease, Schnitzler's syndrome FAPA syndrome (fever, apthous ulcer, stomatitis, pharyngitis, adenitis), Fabry's disease, cholesterol emboli, silicone embolization, Teflon embolization, lymph node infarction, sickle cell disease vasoocclusive crisis, anhidrotic ectodermal dysplasia, cyclic neutropenia, Brewer's yeast ingestion, Hamman-Rich syndrome, Milk-protein allergy, hypersensitivity pneumonitis, extrinsic allergic alveolitis, metal fume fever, polymer fume fever, idiopathic hypereosinophilic syndrome, complex partial status epilepticus, cerebrovascular accident, brain tumor, encephalitis, anomalous thoracic duct, psychogenic fever, habitual hyperthermia, fascititious fever.

Table 2. Conditions associated with bone marrow granuloma ${ }^{36,39}$

\section{Infections}

Tuberculosis

$B C G$ vaccination

Typhoid fever

Brucellosis

Leprosy

Syphilis

Legionnair's disease

Tularemia

Glanders

Viral infections

EBV

Herpes zoster

CMV

HIV

Viral hepatitis

\section{Rickettsial infections} $\mathrm{Q}$ fever

\section{Parasitic infections}

Leishmaniasis

Toxoplasmosis

\section{Fungal infections}

Histoplasmosis

Cryptococcosis

Paracoccidioidomycosis

Coccidioidomycosis

Saccharomyces cerevisiae

\section{Other infections}

Rocky mountain spotted fever

Mycoplasma pneumonia

\section{Dysimmune disorders/Autoimmune}

Farmer's lung

Felty syndrome

Systemic lupus erythematosus

Primary biliary cirrhosis

Rheumatoid arthritis

Sarcoidosis

Drug

Procainamide

Ibuprofen

Phenylbutazone

Dilantin

Indomethacin

Chlorpropamide

Allopurinol

Malignant diseases (with or without tumorous bone marrow involvement) Hodgkin disease

NHL including acute lymphoblastic leukemia

Mycosis fungoides

Multiple myeloma

Metastatic carcinoma

Miscellaneous

Syndrome of marrow and lymph node granuloma

Uveitis, and reversible renal failure.

Berylliosis 


\section{2 b.6. Chronic inflammation with amyloidosis}

In association with chronic inflammation or in dysimmune disease amyloid deposits on vessels walls or in the interstitium has been described. Congo red stain and thioflavin-T-Test are useful in detection of amyloid. Plasma cells are often present around the vessels or around the interstitial amyloid deposits. ${ }^{36}$

\section{Bone Marrow Manifestations in Neoplastic Conditions}

Bone marrow may be primary site for hematological malignancies or may be infiltrated by tumors secondarily that metastasize via blood stream or may show various nonmetastatic effects of distant tumors.

Primary malignancies involving bone marrow are leukemias, myeloproliferative and lymphoproliferative disorders, MDS, histiocytic neoplasms, systemic mast cell diseases and myeloma. MDS is a rare condition which can present with FUO. Prolonged and profound monocytosis in elderly patients with FUO, MDS needs to be ruled out. ${ }^{14}$

Bone marrow involvement is present in Hodgkin lymphoma in $5 \%-15 \%$ cases at the time of presentation. Although any tumor that metastasizes through blood can give rise to bone marrow metastasis, common tumors that metastasize in bone marrow, in adults, are carcinoma of prostate, breast and lung. In children, they are neuroblastoma, rhabdomyosarcoma, Ewing sarcoma and retinoblastoma. Other metastatic tumors include ganglioneuroblastoma in children and melanoma, nephroblastoma, hepatoblastoma and carcinoid tumors. 3\% to $4 \%$ of gastrointestinal malignancies metastasize to bone marrow. Carcinoma of the stomach and colon are the most likely to involve bone marrow. Small cell carcinoma of the lung commonly involves the bone marrow. ${ }^{36}$

Some lymphomas and carcinomas can manifest as leukemoid pattern of marrow and hemophagocytosis in marrow, however, tumor by itself may not involve the marrow. ${ }^{36}$ Bone marrow cytology in metastatic tumors

Metastatic tumor cells are generally concentrated in various sized clusters towards the thin edge and lateral aspects of the smear. Sometimes the bone marrow aspirate is completely or partially necrotic. Malignant cells are usually larger than hematopoietic cells other than megakaryocytes except in cases of small cell tumors in children. Cell outlines may be indistinct or may appear smudged. Some of the cells are multinucleated. Melanoma cells may contain melanin pigment. Malignant cells are usually present in tight clumps. Metastatic adenocarcinoma can be diagnosed on the basis of the formation of glands, presence of signet ring cells and/ or the presence of mucin in cytoplasm. ${ }^{36}$ Leukoerythroblastic feature is frequently seen in peripheral smear. It is not usually possible to predict the tissue of origin from bone marrow aspiration. ${ }^{36}$

\section{Bone marrow histology in metastatic tumors}

Metastatic tumors in bone marrow may be focal, diffuse, or a mixture of both. There may be small foci of tumor or tumor may replace total hematopoietic elements. The bone marrow adjacent to metastatic tumor may be normal or may show nonspecific features such as hyperplasia, increased eosinophils, lymphocytes, granulocytes, or plasma cells. Reticulin and collagen fibers are commonly present. ${ }^{36}$ There may be destruction of bony trabeculae with osteopenia and increased osteoclastic activity. Less frequently, metastasis induces osteoblastic, osteosclerotic reactions with thickened and irregularly shaped bone trabeculae. Myelofibrosis is a common feature to most metastatic tumors. Metastatic disease is most common cause of necrosis in bone marrow. ${ }^{36}$

\section{Non-metastatic effects of cancer on bone marrow morphology}

Patients with cancer but without bone marrow metastasis may show a variety of hematological abnormalities such as hyperplasia of hematopoietic elements; and interstitium may show fibrosis, congestion, or edema. Marrow may show increased iron store or gelatinous transformation. ${ }^{36}$

\section{CONCLUSION}

A combined bone marrow morphology and BMC helps in the arriving at the diagnosis of many but not all the cases of FUO. Many conditions such malignancies, infections and immune disease modify the cellular and interstitial component of marrow which can be studied in bone marrow aspiration and biopsy. BME in FUO is not only used to diagnose the specific disease, but also provides diagnostic clues for the further evaluation by providing important morphological details.

\section{REFERENCE}

1. Arnow PM, Flaherty JP. Fever of Unknown Origin. The Lancet 1997;350: I575-80.

2. Petersdorf RG. Fever of Unknown Origin, An old friend revisited. Arch Intern Med 1992;152:21-2.

3. Laing RBS. Fever and Pyrexia of Unknown Origin. Medicine 2001;29:24-6.

4. Jayram BM, Kanthraj L, Gopinath R, Nagraj BS. Pyrexia of unknown origin. Natl Med J India 1996;9:28-31.

5. Volk EE, Miller ML, Kirkley BA, Washington JA. The diagnostic usefulness of bone marrow cultures in patients with Fever of Unknown Origin. Am J Clin Pathol 1998;110:150-3.

6. Root RK, Petersdorf RG. Fever of Unknown Origin. In: Root RK, Waldvogel F, Corey L, Stamm WE, (eds). Clinical Infectious Diseases. A Practical approach. Oxford Medical Publications:New York;1999.pp459-69.

7. Kohno H, Yamashiro S, Ikeda $\mathrm{N}$ et al. An analysis of Fever of Unknown Origin defined by newly proposed practical criteria. A prospective study of 56 cases. Kansenshogaku-Zasshi 1999;73:62-9. 
8. Durack DT, Street AC. Fever of Unknown Origin-reexamined and redefined. Curr Clin Topics Infect Dis 1991;11:35-51.

9. Knockaert DC, Vanneste LJ, Bobbaers HJ. Recurrent or Episodic Fever of Unknown origin. Review of 45 cases and Survey of the Literature. Medicine 1993;72:184-96.

10. de Kleijn EM, Vandenbroucke JP, van der Meer JW. Fever of Unknown Origin (FUO)-I. A prospective multicenter study of 167 patients with FUO, using fixed epidemologic entry criteria. Medicine 1997;76:392-400.

11. Knockaert DC, Vanneste LJ, Vanneste SB, Bobbaers HJ. Fever of Unknown Origin in the 1980s: An update of the Diagnostic Spectrum. Arch Intern Med 1992;152:51-5.

12. Souhami RL. Cancer: Clinical feature and management. In: Weatherall DJ, Ledingham JGG, Warrel DA, (eds). Oxford Textbook of Medicine, Vol-I. 2nd edn. Oxford Medical Publications: New York;1996.pp 240-53.

13. Gelfand JA. Fever of Unknown Origin. In: Braunwald E, Fauci AS, Kasper DL, Hauser SL, Longo DL, (eds). Harrison's Principles of Internal Medicine, Vol-I. 15th edn. McGraw-Hill: New York: 2001. pp 804-7.

14. Cunha BA, Hamid N, Krol V, Eisenstein L. Fever of unknown origin due to preleukemia/myelodysplastic syndrome: the diagnostic importance of monocytosis with elevated serum ferritin levels. Heart Lung 2006;35:277-82.

15. Hammerstrom J. Bone marrow aspiration. Use and benefits. TidsskrNor-Laegeforen. 1992;112:909-11.

16. Hot A, Jaisson I, Girard C et al. Yield of bone marrow examination in diagnosing the source of fever of unknown origin. Arch Intern Med 2009;169:2018-23.

17. de Kleijn EM, van Lier HJ, van der Meer JW. Fever of unknown origin (FUO). II. Diagnostic procedures in a prospective multicenter study of 167 patients. The Netherlands FUO Study Group. Medicine 1997;76:401-14.

18. Akpek G, Lee SM, Gagnon DR, Cooley TP, Wright DG. Bone marrow aspiration, biopsy, and culture in the evaluation of HIV-infected patients for invasive mycobacteria and histoplasma infections. Am J Hematol 2001;67:100-6.

19. Kumar R, Kalara SP, Kumar H, Anand AC, Madan H. Pancytopenia-A six year study. J Assoc Physicians India 2001;49:1078-81.

20. Esposite AL, Gleckman RA. A diagnostic Approach to the Adult with Fever of Unknown Origin. Arch Intern Med 1979;139:575-9.

21. Khodke K, Marwah S, Buxi G, Yadav RB, Chaturvedi NK. Bone marrow examination in cases of pancytopenia. J Indian Acad Clin Med 2001;2:55-8.

22. Mouallem M, Matetzky S, Raichlin E, Farfel Z. A man with prosthetic valve, anemia, fever and splenomegaly. Lancet 1996;348:1216.

23. Singh KJ, Ahluwalia G, Sharma SK, Saxena R, Chaudhary VP, Anant
M. Significance of Hematological Manifestations in patients with tuberculosis. J Assoc Physicians India 2001;49:788-94.

24. Beris P, Waldvogel FA. Hematologic alterations in Infectious disease patients. In: Root KR, Waldvogel F, Corey L, Stamm WE,(eds). Clinical Infectious Diseases. A Practical approach. Oxford Medical Publications:New York;1999. pp121-31.

25. Brook MG, Ayles H, Harrison C, Rowntree C, Miller RF. Diagnostic utility of bone marrow sampling in HIV positive patients. Genitourin Med 1997;73:117-21

26. Benito $\mathrm{N}$, Núñez $\mathrm{A}$, de Górgolas $\mathrm{M}$ et al. Bone marrow biopsy in the diagnosis of fever of unknown origin in patients with acquired immunodeficiency syndrome. Arch Intern Med 1997;157:1577-80.

27. Bartl R, Frisch B, Baumgart R. Morphologic classification of the Myelodysplastic syndrome (MDS):combined utilization of bone marrow aspirates and trephine biopsies. Leuk Res 1992;16:15-33.

28. Jha A, Sarda R, Gupta A, Talwar OP. Bone Marrow Culture Vs Blood Culture in FUO. J Nepal Med Assoc 2009;48:135-8.

29. Wain J, Bay PVB, Vinh H, et al. Quantitation of bacteria in bone marrow from patients with typhoid fever: Relationship between counts and clinical features. J Clin. Microbiol 2001;39:1571-6.

30. Hussong J, Peterson LR, Warren JR, Peterson LAC. Detecting Mycobacterium avium complex infections in HIV - positive patients. The usefulness of bone marrow trephine biopsy specimens, aspirate cultures, and blood cultures. J Clin Pathol 1998;110:806-9.

31. Farhi DC, Mason UG, Horsburg CR. The Bone marrow in Disseminated Mycobacterium avium-intracellulare Infection. Am. J Clin Pathol 1985;83:463-468.

32. Deepak S, Bronson SG, Sibi, Joseph W, Thomas M. Brucella isolated from bone marrow. J Assoc Physicians India 2003;51:717-8.

33. Chattrjee KD. Parasitology (Protozoology and Helminthology) in relation to clinical medicine.12th ed. Chattterjee medical publishers:Calcutta;1981.54-65pp.

34. Shenoi U, Pai R, Pai U, Nandi GK, Adhikari P. Microfilaria in Bone marrow aspiration smears. Acta Cytologica 1998;42:815-6.

35. Bain BJ, Clark DM, Lampert IA, editors. Bone Marrow Pathology. 2nd ed. Blackwell Science:Oxford;1996: 287-306pp.

36. Diebold J, Molina T, Camilleri-Broet S, Tourneau A le, Audouin J. Bone marrow manifestations of infections and systemic diseases observed in bone marrow trephine biopsy. Histopathology 2000;37:199-211.

37. McKenna RW, Risdall RJ, Brunning RD. Virus associated hemophagocytic syndrome. Hum Pathol 1981;12:395-39.

38. Band $\mathrm{P}$, Khan $\mathrm{AB}$. Bone marrow changes in Kala-Azar. J Assoc Physicians India 1991;4:41-3

39. Bodem CR, Hamory BH, Taylor HM, Kleopfer L. Granulomatous bone marrow disease. A review of the literature and clinicopathologic analysis of 58 cases. Medicine 1983;62:373-83. 